\title{
Hydrogen Chloride Optical Gas Standards (OGS) at PTB
}

\author{
Javis Nwaboh, Zhechao Qu, Olav Werhahn, Volker Ebert \\ Physikalisch-Technische Bundesanstalt (PTB), 38116 Braunschweig, Germany, \\ Corresponding Author: javis.nwaboh@ptb.de
}

\begin{abstract}
Summary:
Accurate and reliable measurements of hydrogen chloride $(\mathrm{HCl})$ are required in applications such as biomethane quality control, clean room monitoring or stack emissions monitoring. In order to perform $\mathrm{HCl}$ measurements, standardized measurement methods as well as accurate reference gases are required to calibrate typical $\mathrm{HCl}$ measurement instruments. However, there is a lack of SI-traceable $\mathrm{HCl}$ reference gases and reliable quality control test methods for many of these applications, once future more challenging limit values have been put into force by new regulations to come. To this end, PTB is developing optical gas standards (OGS), e.g., for $\mathrm{HCl}$ quantifications in those applications and to complementing existing gaseous reference standards. In this paper, we report on the HCl-OGSs developed in PTB for different applications.
\end{abstract}

Keywords: Metrology, Gas Analysis, Optical Gas Standard (OGS), TILSAM, dTDLAS.

\section{Introduction}

Gaseous hydrogen chloride $(\mathrm{HCl})$ poses severe health effects when inhaled and can form corrosive hydrochloric acid on surfaces when it meets water. These properties accelerate the need for accurate $\mathrm{HCl}$ detection e.g. for quality control measurements in biomethane, airborne molecular contaminations monitoring in clean rooms or stack gas emissions [1]. Accurate $\mathrm{HCl}$ measurements typically require validated test methods [1]. Reliable test methods are lacking for $\mathrm{HCl}$ quantifications in biomethane, an energy gas that is seen to replace parts of the fossil natural gas sources in existing grids [1]. For stack emissions, $\mathrm{HCl}$ measurements are referred to the $\mathrm{HCl}$ - standard reference method described in EN 1911 (on the determination of mass concentration of gaseous chlorides). EN 1911 is based on wet chemistry. Hence, a gas sample is extracted, particle-filtered and dried and then dissolved in water, to analyze the $\mathrm{Cl}$-ion concentration in the liquid. This sampling procedure can easily lead to systematic deviations. $\mathrm{HCl}$ amount factions in biomethane and clean room air are required to stay at low $\mu \mathrm{mol} / \mathrm{mol}$ to the $\mathrm{nmol} / \mathrm{mol}$ levels. $\mathrm{HCl}$ sensor calibration requires calibration gas standards in the same range. However, generation and provision of gaseous reference materials traceable to the international system of units (SI) has proven to be difficult [1], e.g. there are no calibration and measurements capabilities (CMCs) reported for $\mathrm{HCl}$ amount fractions below $10 \mu \mathrm{mol} / \mathrm{mol}$ (https://kcdb.bipm.org/). Only a few National Metrology Institutes (NMIs) have CMCs for $\mathrm{HCl}(10-1000 \mu \mathrm{mol} / \mathrm{mol})$ in $\mathrm{N}_{2}$. For $\mathrm{HCl}$ in more complex gas matrices (e.g. biomethane), there are no CMCs available at all. Optical gas standards [3], [4] provide the option to be mandated as test methods for $\mathrm{HCl}$ measurements in the above mentioned applications. Due to the $1^{\text {st }}$ principles measurement approach, OGSs do not require calibration with a calibration gas mixture, and therefore can be used to complement gaseous reference standards in the low $\mu \mathrm{mol} / \mathrm{mol}$ down to the $\mathrm{nmol} / \mathrm{mol}$ levels [2, 3]. Furthermore, an OGS can also be used for $\mathrm{HCl}$ measurements directly in the field in situ. In this paper, we present $\mathrm{HCl}$ OGS instruments compliant with the TILASM method [4] and developed or currently being developed at PTB.

\section{An Optical Gas Standard (OGS)}

The measurement technique employed in an OGS instrument (see Fig. 1) is direct tunable diode laser absorption spectroscopy (dTDLAS) [2-3], [5-6]. dTDLAS is a variant of TDLAS that combines TDLAS with a $1^{\text {st }}$ principles data evaluation approached to derive absolute gas species amount fractions that are directly traceable to the SI. An OGS laser spectrometer is thus similar to the National institute of Science and Technology (NIST) ozone standard reference Photometer (SRP). Employing the BeerLambert law on a continuously scanned diode laser spectrometer and deriving the line area (Aline) underneath an absorption line, a SI- 
traceable $\mathrm{HCl}$ amount fraction is inferred. Figure 1 shows an $\mathrm{HCl}$ absorption profile in $\mathrm{N}_{2}$ matrix gas measured at a wavelength of about $3.6 \mu \mathrm{m}$ and evaluated according to equation 1 as resulting a $518.4 \mu \mathrm{mol} / \mathrm{mol} \mathrm{HCl}$ amount fraction without the need to calibrate the instrument with a gaseous calibration standard [3], [4]. Repeated measurements are shown in Fig. 2.

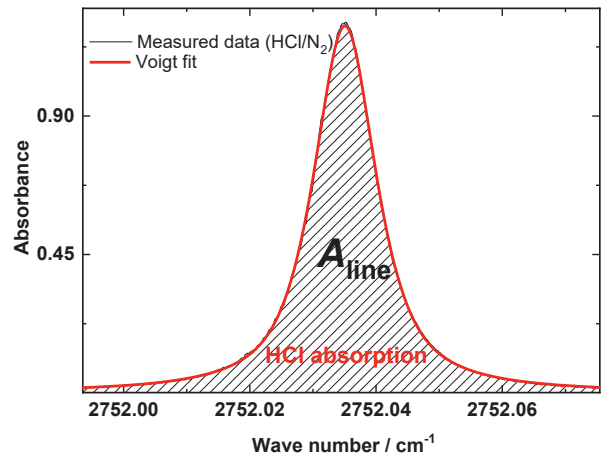

Fig. 1: Typical $\mathrm{HCl}$ single line absorbance spectrum measured by an OGS instrument operated at $3.6 \mu \mathrm{m}$.

$$
x_{\text {species }}=\frac{k_{B} \cdot T}{S_{T} \cdot L \cdot p_{\text {total }}} \cdot A_{\text {line }}
$$

For an $\mathrm{HCl}$ OGS, ensuring that all input quantities on the right-hand side of Eq. 1 are SItraceable, the $\mathrm{HCl}$ amount fraction (concentration) $x_{\mathrm{HCl}}$ is directly traceable to the SI. The quantities $k_{\mathrm{B}}$ being the Boltzmann constant, $S_{T}$ the line strength of the probed molecular transition at gas temperature $T, L$ the path length of the light beam transmitted through the absorbing medium and $p_{\text {total }}$ the total gas pressure. The SI-traceability of these input quantities is ensured similarly to references $[2,3,4]$.

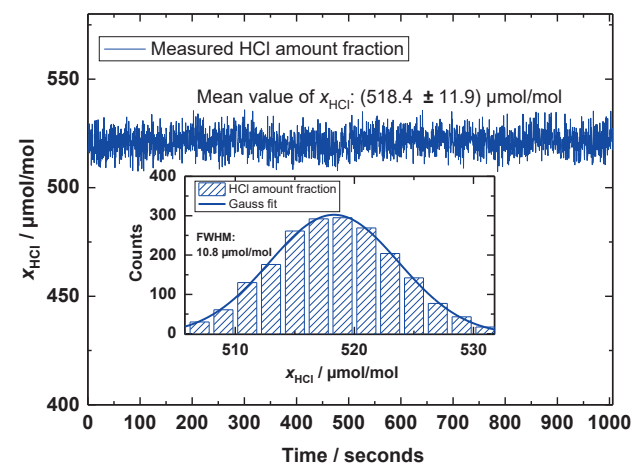

Fig. 2: $\mathrm{HCl}$ amount fraction (repeated measurements) as a function of time. Inset: A histogram depicting a normal distribution of the results and depicting the performance of an OGS.

Delivering SI-traceable amount fractions, an OGS is a "calibration free" instrument and can therefore be used as described to complement (support and use in the place of) calibration gases both in the lab and in the field, especially for sticky and reactive gases such as $\mathrm{HCl}, \mathrm{H}_{2} \mathrm{O}$ and $\mathrm{NH}_{3}$.

\section{Summary}

Table 1 lists some details of $\mathrm{HCl}$ OGS systems developed/currently being developed at PTB. The OGS-Biomethane instrument is currently being used in a bilateral comparison with the Korean NMI, KRISS. Furthermore, the instrument will be employed in a CCQM key comparison of $\mathrm{HCl}$ in air, planned to be run at a $30 \mu \mathrm{mol} / \mathrm{mol}$ level.

Table 1: Summary of $\mathrm{HCl}$ OGS systems developed/currently being developed at PTB.

\begin{tabular}{|l|c|c|l|}
\hline $\begin{array}{l}\text { OGS sys- } \\
\text { tems of } \\
\text { PTB clus- } \\
\text { ter }\end{array}$ & $\begin{array}{l}\text { Targeted } \\
\text { range / } \\
\mu \text { mol/mol }\end{array}$ & $\begin{array}{l}\text { Rel. com- } \\
\text { bined } \\
\text { uncertainty } \\
(\boldsymbol{k}=1) / \%\end{array}$ & Gas Matrix \\
\hline $\begin{array}{l}\text { OGS- } \\
\text { Biomethane }\end{array}$ & $\begin{array}{c}0.025- \\
500\end{array}$ & 2.3 & $\begin{array}{l}\mathrm{CH}_{4} \text { (or } \\
\text { biomethane), } \\
\mathrm{N}_{2} \text {, air }\end{array}$ \\
\hline OGS-Stack & $0.05-$ & $2-4$ & $\begin{array}{l}\text { Flue gas } \\
\text { (with high } \\
\left.\mathrm{CO}_{2}, \mathrm{H}_{2} \mathrm{O}\right)\end{array}$ \\
\hline & 100 & & Air (or $\left.\mathrm{N}_{2}\right)$ \\
\hline OGS-AMC & $0.001-1$ & $2-4$ & \multicolumn{2}{|c|}{$2-4$ conformity assessments. }
\end{tabular}

OGS-Biomethane: $\mathrm{HCl}-\mathrm{OGS}$ instrument for bio-CH4 conformity asse

OGS-Stack: HCl-OGS instrument for stack emissions monitoring.

\section{Acknowledgement}

Parts of this work was supported by the European Metrology Programme for Innovation and Research (EMPIR). The EMPIR initiative is cofunded by the European Union's Horizon 2020 research and innovation programme and the EMPIR Participating States. The authors would also like to acknowledge the support from current and past members of the PTB working group 'Spectrometric Gas Analysis'

\section{References}

[1] EMPIR project Biomethane (http://empir.npl.co.uk/biomethane/), IMPRESS 2 (http://empir.npl.co.uk/impress/), MetAMCII, (http://empir.npl.co.uk/metamcii/).

[2] J. A. Nwaboh, Z. Qu, O. Werhahn and V. Ebert, "Interband cascade laser-based optical transfer standard for atmospheric carbon monoxide measurements", Applied Optics, 56, E84-E93 (2017).

[3] B. Buchholz, N. Böse and V. Ebert, "Absolute validation of a diode laser hygrometer via intercomparison with the German national primary water vapor standard", Applied Physics B, 116, 883899 (2014).

[4] O. Werhahn, J.C. Petersen (eds.), 2010, TILSAM technical protocol V1_2010-09-29. Available from: http://www.euramet.org/fileadmin/docs/projects/934 METCHEM_Interim_Report.pdf.

[5] Ż. Qu, J. Nwaboh, O. Werhahn and V. Ebert, "Towards a dTDLAS-Based Spectrometer for Absolute $\mathrm{HCl}$ Measurements in Combustion Flue Gases and a Better Evaluation of Thermal Boundary Layer Effects", Flow, Turbulence and Combustion, Sept. 2020, doi: 10.1007/s10494-020-00216-z.

[6] A. Pogany, S. Wagner, O. Werhahn and V. Ebert, "Development and Metrological Characterization of a Tunable Diode Laser Absorption Spectroscopy (TDLAS) Spectrometer for Simultaneous Absolute Measurement of Carbon Dioxide and Water Vapor", Applied spectroscopy, 69, 2, 257-268 (2015). 